\title{
Short-Term and Long-Term Effects of Vocal Distortion on Song Maintenance in Zebra Finches
}

\author{
Gerald E. Hough II and Susan F. Volman \\ Department of Evolution, Ecology and Organismal Biology, Ohio State University, Columbus, Ohio 43210-1293
}

Adult zebra finch song is irreversibly altered when birds are deprived of correct feedback by deafening or denervation of the syrinx. To clarify the role of feedback in song maintenance, we developed a reversible technique to distort vocal output without damaging the auditory or vocal systems. We implanted flexible beads adjacent to the syrinx to alter its biomechanics. Immediate song aberrations included low volume, frequency shifts, missing harmonics, and production of click-like syllables. After a few weeks, seven of nine birds stopped producing some syllables. In six of these birds, the gaps left by the silenced syllables gradually shortened, and the lost syllables did not return when beads were removed 16 weeks after treatment began. The nondeleted syllables of all birds regained their preimplant morphology, insofar as could be detected, within $9 \mathrm{~d}$ after bead removal. In four other birds, we removed the beads as soon as syllables were deleted, when the silent intervals were still full length. In these birds, all deleted syllables returned within 1 week. Our results indicate that both silenced syllables and syllable morphology can recover as long as the song's temporal structure is maintained, but once altered, changes in the song sequence can be permanent. A hierarchical organization of the song production system has recently been described (Margoliash, 1997). Reversible disruption of song production by our method appears to permanently alter the higher levels of the system that encode song sequence, but not the lower levels that encode individual syllable structure.

Key words: zebra finch; auditory feedback; vocal distortion; song; HVc; songbirds; vocal learning
Songbirds learn their vocalizations by memorizing the songs of conspecific individuals heard during early life. Young birds then use this memory to guide their own vocal production through auditory feedback to match the memorized songs (Konishi, 1965; Marler, 1970). As song improves, it becomes "crystallized" and stereotyped. After song crystallization, birds such as the zebra finch (Taeniopygia guttata) (Price, 1979), white-crowned sparrow (Zonotrichia leucophrys) (Konishi, 1965), and chaffinch (Fringilla coelebs) (Nottebohm, 1968) maintain the same crystallized songs throughout their lives, although with some seasonal instability (Tramontin and Brenowitz, 2000; Hough and Volman, 2000). The phenomenon of song crystallization suggests that these birds develop a fixed motor program that generates stereotyped vocalizations (Nottebohm, 1968).

Several techniques have been used in attempts to determine the importance of vocal feedback in the maintenance of adult crystallized song. In zebra finches, song change has been observed after birds were deafened by cochlear removal (Nordeen and Nordeen, 1992; Scott et al., 2000), after their vocalizations were distorted by tracheosyringeal (nXIIts) nerve transections (Williams and McKibben, 1992; Williams and Mehta, 1999), and while they were being subjected to delayed syllable playback during singing (Leonardo and Konishi, 1999). Birds of a closely related

Received Oct. 13, 2000; revised Nov. 21, 2001; accepted Nov. 19, 2001.

This work was funded by National Institutes of Health Grant MH47330 (S.F.V.) and an Osborne Fellowship (G.E.H.). We thank K. C. Schuett for animal care. We also thank Jill Soha, Abbot S. Gaunt, and Sandra L. L. Gaunt for comments on this manuscript.

Correspondence should be addressed to G. E. Hough II, Department of Psychology, Bowling Green State University, Bowling Green, OH 43403. E-mail: ghough@bgnet.bgsu.edu.

S. F. Volman's present address: National Institutes of Health, National Institute on Drug Abuse, 6001 Executive Boulevard, Room 4282 MSC 9555, Bethesda, MD 20892.

Copyright (C) 2002 Society for Neuroscience $\quad 0270-6474 / 02 / 221177-10 \$ 15.00 / 0$ species, the Bengalese finch (Lonchura striata domestica), have also been deafened by cochlear removal (Okanoya and Yamaguchi, 1997; Woolley and Rubel, 1997; Scott et al., 2000) and partially deafened by hair cell destruction (Woolley and Rubel, 1999). In all but the study using a noninvasive technique (Leonardo and Konishi 1999), in which gradual song recovery occurred after treatment ended, permanent changes in song were induced by these manipulations. These changes included loss or addition of syllables, elisions of introduced silent intervals, degradation of syllable structure, and other large-scale changes. However, some recovery of syllable morphology was observed in the nerve transection experiments.

Deafening and nerve transections not only prevent or alter auditory and/or proprioceptive feedback, but they may also induce neuronal changes in brain areas that subserve song production. Because the song system has major auditory inputs, deafening by cochlear removal may cause anterograde degeneration or synaptic changes; this treatment has been shown to retard neuronal turnover in the song system (Wang et al., 1999). Nerve transections may produce retrograde synaptic reorganization or neuronal degeneration of motor neurons or their afferents.

To assess the effects of changing song feedback without introducing possible secondary neuronal changes, we developed a technique to mechanically obstruct syringeal function that distorted the birds' vocalizations while preserving all anatomical connections to the song system, and which could be easily removed. We used this treatment to ask whether song changes were permanent or temporary and what aspects of the altered song would be recovered after normal syringeal function was restored. The results of this study have specific implications for understanding the normal role of vocal feedback in song production and the neural basis of song change when feedback is disrupted. 
A preliminary report has been published previously (Hough and Volman, 1996).

\section{MATERIALS AND METHODS}

Subjects. Subjects were adult male zebra finches at least $129 \mathrm{~d}$ of age (mean, $475 \mathrm{~d}$; range, 129-1815 d), reared in our captive colony. We maintained birds on a 16:8 light/dark cycle. Finch seed, grit, and water were provided ad libitum, supplemented weekly with lettuce and ground hard-boiled eggs. Birds were housed in visual and acoustic contact with other individuals throughout song development and during the experiment. At the start of each experiment, every bird was in adult plumage and singing crystallized song.

We recorded the song of each bird before the experimental treatments to confirm that the song motifs were stable and contained at least four easily distinguished syllables. A syllable is defined as a single continuous vocalization separated from other vocalizations by either a short silent interval or by a major change in harmonic structure, whereas a motif is defined as a repeated set of syllables occurring in the same order (Sossinka and Böhner, 1980). Birds sang motifs in a highly stereotyped manner, with no discernable variation in syllable order across renditions.

We divided birds into three treatment groups. Nine birds were used in a 16 week distortion experiment (long-term bead birds). Five birds were used as long-term controls (sham bead). Finally, four birds were used in a short-term distortion experiment (short-term bead). There was no evidence that any procedure used in this study caused sustained stress or discomfort. We performed these experiments under a protocol approved by The Ohio State University's Institutional Laboratory Animal Care and Use Committee.

Treatments. We fashioned flexible beads, $\sim 1-2 \mathrm{~mm}$ in diameter, of dental impression material (Permlastic; Kerr, Inc.). This material was selected because of its flexibility, ease of formation, and biological neutrality. A short piece of silk suture thread was tied around the bead to facilitate removal in all but the first two birds. Neither the bead nor thread adhered to any tissue with which it came into contact, even after 4 months.

Birds were anesthetized before surgery with $0.05 \mu \mathrm{l}(\sim 4 \mathrm{ml} / \mathrm{kg})$ of a pentobarbital-chloral hydrate anesthetic similar to Equithesin, which resulted in $\sim 15 \mathrm{~min}$ of deep anesthesia. If a bird reacted to any portion of the insertion procedure, it received another $0.02 \mu \mathrm{l}$ of anesthetic, and the procedure was halted until anesthesia resumed (no response to a toe-pinch).

We made a rostrocaudal incision in the skin of the throat using microscissors and moved aside the fat pad (which was kept moist with a saline-soaked tissue) to reveal the underlying interclavicular air sac. The syrinx was clearly visible through a surgery microscope after we punctured the air sac with fine forceps. The bead was inserted with the aid of a modified dental pick into a cavity that lies external to the syrinx between the two medial tympanic membranes and the bronchidesmus (Fig. 1). This cavity is not part of the airway system itself. The bead apparently disturbed the biomechanics of the syrinx by narrowing the airway and applying pressure to the medial labia, which prevented the membranes from vibrating normally (Goller and Larsen, 1997). For a recent review of syringeal function, see Suthers et al. (1999).

Once the bead was in place, we returned the fat pad to its normal position and closed the skin with fine silk sutures. In some birds, we also used Vetbond tissue glue (3M Animal Products, St. Paul, MN) to close the skin if gaps between sutures were large. We were careful to restore the fat pad and close the skin thoroughly because air pressure in the interclavicular air sac is necessary for normal syringeal function in oscine songbirds (Smith, 1979; McDonald, 1989). The entire insertion procedure lasted between 10 and 15 min. Birds usually awoke within minutes of wound closure, and after $\sim 1 \mathrm{hr}$ they were perching normally. We did not observe any adverse effects on behavior or breathing in birds with beads after a few days of recovery.

Long-term distortion. In the long-term bead birds, we removed the bead after 16 weeks. We chose this duration to allow comparison with results of a previous deafening experiment in which substantial song changes were apparent within the first 16 weeks after cochlear removal (Nordeen and Nordeen, 1992). The removal procedure was similar to bead insertion. In all birds, the bead was intact and easily removed by grasping the attached suture thread or the bead itself with forceps and pulling lightly away from the syrinx.

Long-term controls. These birds underwent sham bead insertion and removal. We inserted the bead into the syrinx and then immediately removed it once at the beginning and once at the end of a 16 week period.

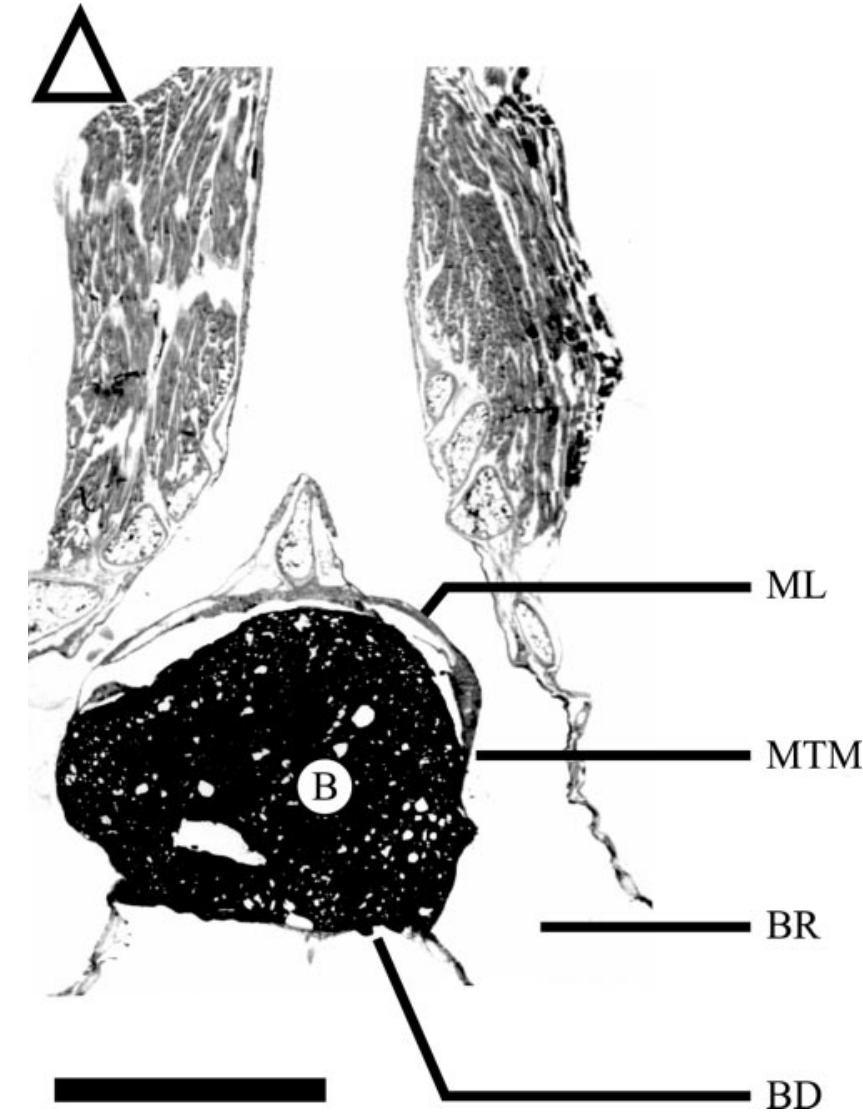

Figure 1. Bead $(B)$ located in the syrinx. The bead contacts the soundproducing structures, the medial labium $(M L)$ and the medial tympanic membrane $(M T M)$, and it is held in place by the bronchidesmus $(B D)$. The bird's vocalizations are distorted, most likely because the close proximity of the bead to the sound-producing structures prevents them from vibrating normally. The pressure of the bead on the ML and MTM also partially occludes the bronchi $(B R)$, which can reduce the volume of the song. The arrowhead points in the rostral direction. Scale bar, $1 \mathrm{~mm}$.

This procedure controlled for any disruptions to song caused by surgery or any damage done to the syrinx by placing a bead in contact with the vibratory membranes. The control birds also provided a baseline of normal song changes that may occur over a 16 week period.

Short-term distortion. As described below, long-term bead treatment resulted in the permanent loss of song syllables that were dropped within 3-10 weeks of bead implantation. To determine whether syllable deletions are permanent as soon as they occur, we also performed a shortterm bead study. In this group, we monitored the birds' vocalizations weekly after bead implantation, and we removed the beads as soon as syllables were dropped. Seven birds were originally prepared for the short-term study, but we report here only on four birds that dropped syllables within 1 month after bead insertion (one died during removal surgery, and two did not drop syllables within 1 month).

Song recording and analysis. We recorded the vocalizations of each bird in a separate isolation chamber (model IAC-1; Industrial Acoustics). A Marantz PMD-222 audiocassette recorder connected to a Toa K-1 microphone inside the chamber automatically recorded the first $45 \mathrm{~min}$ of morning songs after the lights turned on. In cases where birds failed to sing spontaneously, they were coaxed to sing by temporarily introducing a female in a separate sound-attenuating compartment (a glass jar with a foam rubber stopper), which prevented the female's calls from overlapping the male's song. Both directed and undirected songs were analyzed because they are composed of identical motifs and differ only slightly in tempo (Sossinka and Böhner, 1980).

Before treatment, we recorded at least 10 songs from each bird. If a bird did not sing 10 songs, we set up the recorder for the next day. We recorded each experimental bird weekly during the treatment (although in a few cases they did not sing during the $2 \mathrm{~d}$ in the recording chamber 
in a given week), several times in the first 3 weeks after bead removal, and occasionally thereafter for up to 2 years. After surgeries, we usually waited 1 week before recording a bird, which allowed the air sac and skin to heal: in birds recorded a few days after air sac puncture, song was low in volume and distorted (even in those birds that had no beads inserted). These effects were mostly gone by 1 week after surgery, although two birds took $9 \mathrm{~d}$ to recover robust vocalizations. We recorded the control birds 1 week before sham implantation and 1 week after sham removal. We examined all of the recordings initially using a Kay Elemetrics DSP Sona-Graph model 5500 at the Borror Laboratory of Bioacoustics at The Ohio State University. Songs were visually inspected for changes in syllable phonation, amplitude, and order. In all cases, we used songs with at least two complete motifs for examining changes in the number of syllables, because some birds optionally drop the final syllables of the last motif sung. Only the syllables from the first motif were used in the morphological analyses.

To quantify the effects of bead treatment on syllable morphology, we calculated spectrogram cross-correlation coefficients of syllables from the long-term bead and long-term control birds using Canary (Cornell Laboratory of Ornithology, Ithaca NY). Cross-correlation analysis compares the amount of overlap between spectral components of two sounds, with differences in frequency, amplitude, and duration resulting in lower correlation values. Although there can be problems with the use of spectrogram cross-correlation on harmonic notes such as those present in zebra finch song (Khanna et al., 1997), none of these arose with our data. For example, we did not observe harmonic shifts equal to the fundamental frequency, which would result in a sound of much higher pitch, but a very high correlation coefficient compared with a syllable shifted to a lesser degree. Other methods for calculating similarity between harmonic vocalizations have been devised (Janik, 1999; Tchernichovski et al., 2000), but because we were comparing easily identified same-syllable elements within individuals, we found that a cross-correlation analysis was appropriate for our purposes.

For the calculations of correlation coefficients, we extracted syllables from three randomly selected exemplars of song recorded in a single session; only the first occurrence of each syllable type was used for analysis. We used two sets of songs for the first analysis: those recorded just before bead or sham insertion, and those recorded within 2 weeks after bead or sham removal. In no case did we have any difficulty identifying the song syllables, in part because we had preselected birds whose songs had distinct syllables, and the treatment produced little change in syllable morphology (see Results). To establish a measure of variability for songs recorded on a single day, we calculated pairwise autocorrelations for each syllable across the three songs, and then averaged all correlation values for the song to obtain a single "autocorrelation score" for each bird (three correlation values per syllable and a range of three to six syllables per bird). Change in syllable morphology caused by treatment was determined by cross-correlating the three replicates of individual syllables recorded before treatment with those recorded after treatment and averaging the correlations across all syllables to obtain a "treatment-correlation score" (nine correlation values per syllable and three to six syllables per bird).

We performed two analyses on the correlation values. The first compared pretreatment autocorrelation scores with post-treatment autocorrelation scores for the long-term bead birds. If song distortion causes an increase in syllable variability, we would expect lower autocorrelation scores for post-treatment syllables relative to pretreatment syllables. The second analysis compared the treatment correlations between the longterm bead and control birds to assess overall changes in syllable structure. If treatment causes a change in the structure of individual syllables, we would expect the treatment cross-correlation scores to be lower in bead birds than in control birds. In both analyses, we compared only those syllables that were present in both prebead and postbead song and used nonparametric statistics to assess differences between groups because of the small sample sizes. All values are reported as mean $\pm \mathrm{SE}$.

We used two methods to assess the sensitivity of cross-correlation analysis to detect differences in morphology. First, each of the 51 prebead syllables from the long-term bead birds was correlated with the 25 prebead syllables from the sham treated birds and the highest value was selected (we used correlations only from across the two groups because three of the bead birds were related and shared song syllables). We then visually inspected the spectrograms of these best-correlation pairs and eliminated seven of them where the paired syllables were not of the same general syllable type. The remaining correlation values for each bead bird were then averaged to compare with the within-bird treatment correlation values described above. Second, we correlated prebead syllables with the same syllables recorded on various days during the long-term bead treatment. We averaged the values across each month of treatment for statistical analysis (nonparametric pairwise comparisons). These measures were used to assess changes in syllable distortion during treatment and as another measure of the sensitivity of the correlation procedure to detect differences in syllable morphology.

To determine whether incorrect feedback affects different types of syllables differently, we compared the relative proportions of syllable types before and after treatment. Syllables were segregated into four morphological groups: harmonic stacks, harmonic sweeps, highfrequency notes, and noisy broadband syllables. These are similar to the groups used by Williams and Staples (1992) and Sturdy et al. (1999).

To assess the effect of bead treatment on temporal properties of song, we measured the change in the silent interval created when syllables were deleted. Interval duration was measured from the start of the amplitude envelope of the syllable preceding a group of deleted syllables to the start of the amplitude envelope of the first syllable after the deleted group. These intervals varied depending on the duration of the syllables measured and the number of syllables dropped. Therefore, for analysis, interval measures were normalized relative to the duration of the interval before treatment.

Histology. We dissected out the syrinx of a bird that died during bead removal in the short-term experiment to visualize the location of the implanted bead in the syrinx. The syrinx was decalcified, embedded in paraffin, cut on a vibratome at a thickness of $100 \mu \mathrm{m}$, and stained with hematoxylin-eosin (Fig. 1). Despite having been in contact with the bead for 4 weeks, the syrinx appeared normal.

\section{RESULTS}

In both the long-term and short-term bead birds, many changes in song occurred immediately after bead insertion, and in some cases note morphology also continued to change over many weeks (Fig. 2). There was a reduction in the number of harmonics and the fundamental frequency of many harmonic notes, and vocalizations were reduced in volume in most subjects. Other more severe distortions included a tendency for harmonic sweeps and noisy syllables to distort into brief, broadband clicks. In all cases, song during treatment sounded unlike normal zebra finch song. All of the syllables were present in some form for the first few weeks after we inserted the bead, but then most birds dropped one or more syllables, leaving, at first, a silent interval in the song motif. In all cases, the deleted syllables were adjacent to each other in the motif sequence, disappeared at the same time (within the limits of our once-a-week recordings), and were missing from every rendition of the song motif. One short-term bird began to repeat the initial syllable of his first motif, and one long-term bird began to repeat the final syllable of his last motif (Fig. 3, right). These song variants are sometimes seen in normal song (and are unlike the stuttering that has been reported in other feedback disruption studies), but in our birds, the repetitions appeared during treatment and disappeared afterward, so we are reporting them as changes caused by treatment.

\section{Long-term distortion}

During treatment, the distortion sometimes varied (Fig. 2), but it did not significantly increase or decrease over time, as measured by cross-correlation with prebead syllables (see Fig. 5). Seven of the nine birds lost syllables from their songs while the bead was in place, and only one bird added a new syllable (Figs. 4a, 5). Birds deleted an average of $1.5 \pm 0.4$ syllables (range $0-4)$ from their songs. Across all birds, 15 of 51 syllables were deleted, and one was added. The five sham-treated birds did not delete any of the 25 syllables collectively present in their songs. Syllables were dropped between weeks one and four by five of the bead birds, and between weeks six and 10 by two birds whose songs were not recorded as frequently. The location of the deleted syllables was 

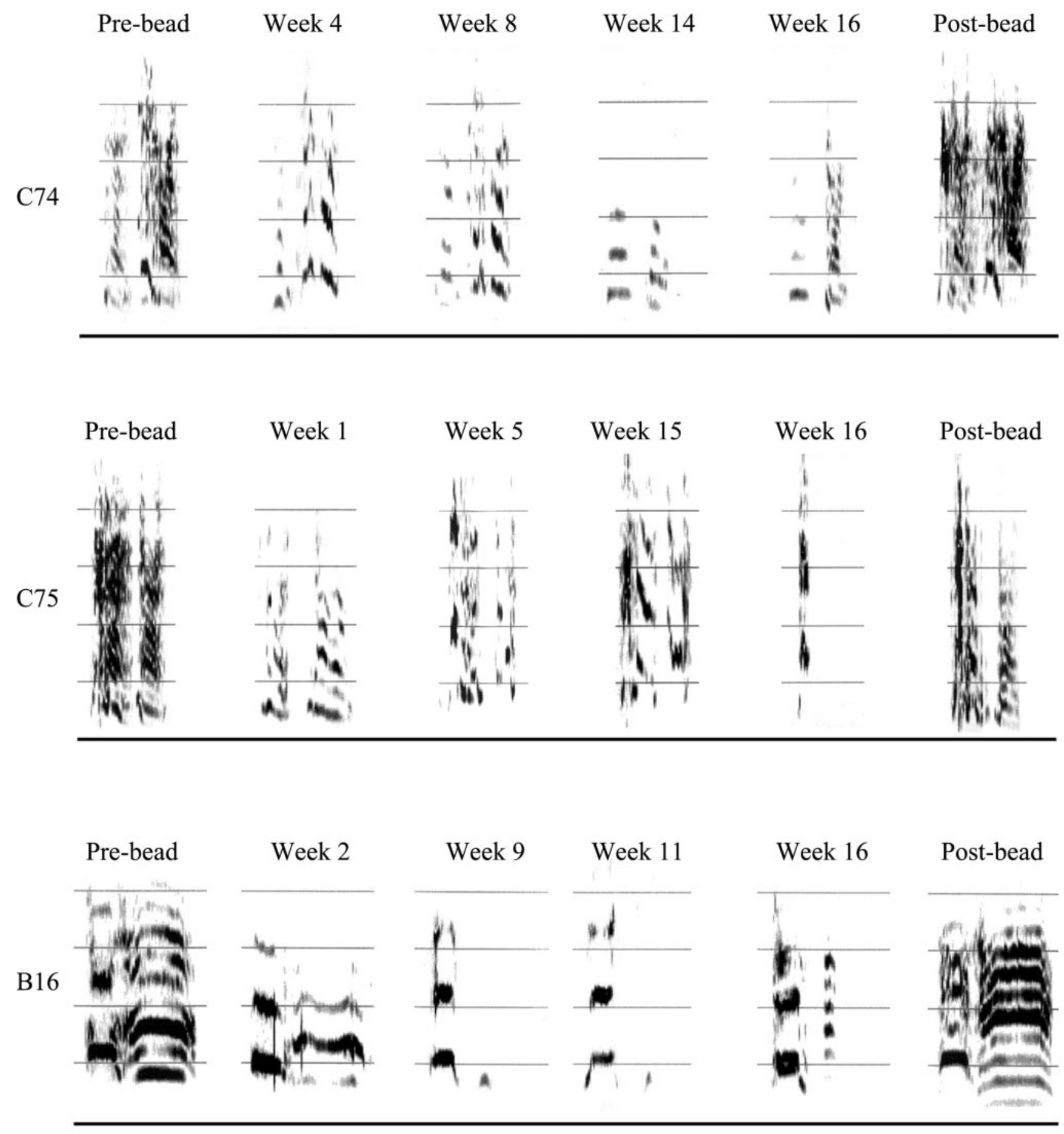

Figure 2. Examples of distortion of individual syllables caused by bead treatment. The first column shows the prebead morphology. Distortions seen here include alteration of harmonic structure and truncation. In addition, the fundamental frequency of B16's syllables (bottom panel) decreased. The last column shows the syllable morphology within the first 2 weeks after the bead was removed. Scale bar, $0.5 \mathrm{sec}$; vertical axis, $2 \mathrm{kHz}$.

variable, occurring at the start (in one bird), in the middle or at the end of the song. By the end of treatment, the intersyllable intervals, where the dropped syllables had been, had shortened in six of the seven birds to a mean of $66 \%$ of their former duration (Fig. 6).

No syllable types appeared to be particularly vulnerable to deletion, because we did not detect a change in the relative numbers of syllable types (harmonic stack, sweep, high-frequency, and noisy syllables) before and after bead treatment $\left(\chi^{2}\right.$ test, $\mathrm{df}=$ $3, \chi^{2}=1.667$, NS). Birds with more variable songs before treatment were more likely to drop syllables: the number of deleted syllables was significantly negatively correlated with the spectrographic autocorrelations of pretreatment syllables (Spearman's rho; $N=9 ; r=-0.821 ; p=0.007$ ) (Fig. 7). However, the prebead variability of syllables that were dropped was not larger than the variability of syllables that were retained (Mann-Whitney $U$ test; 
a

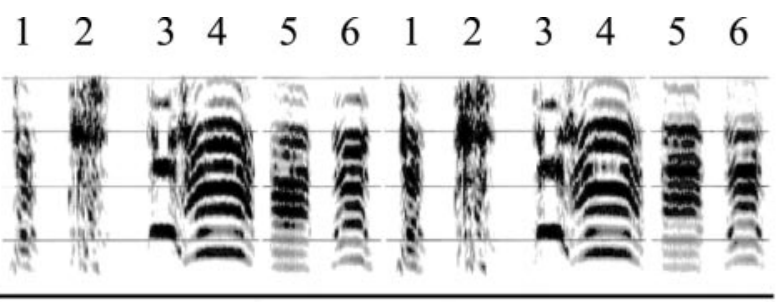

$\mathrm{b}$

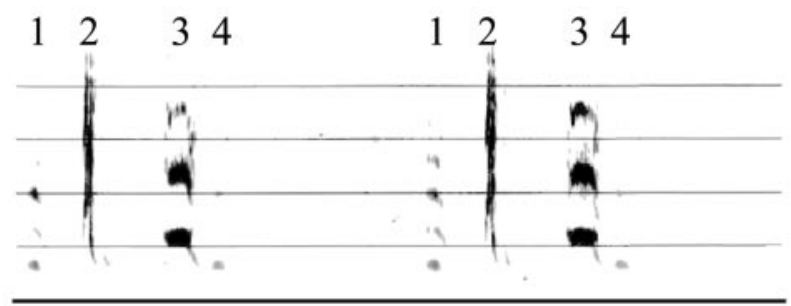

C

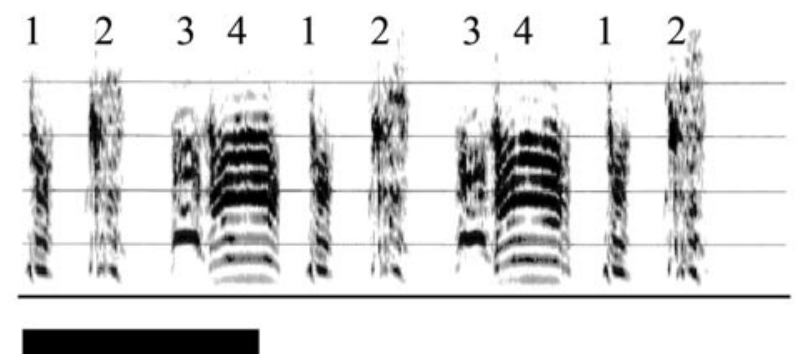

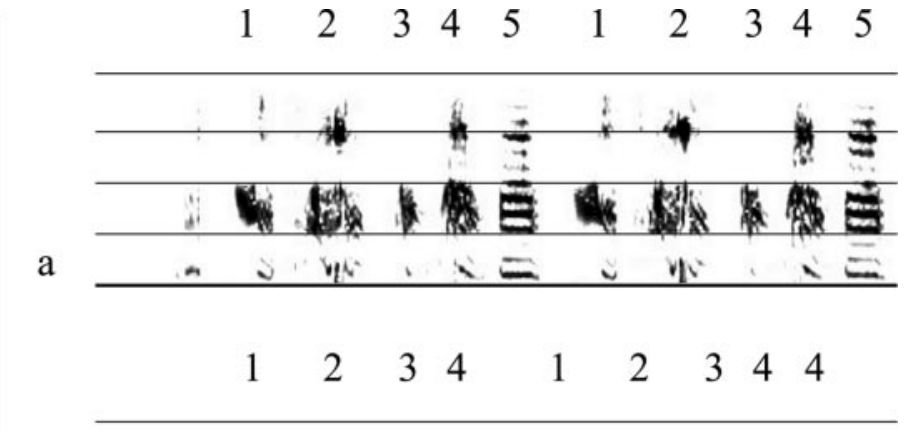

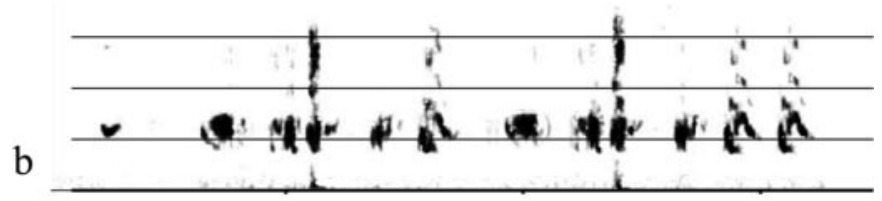

$\begin{array}{llllllll}1 & 2 & 3 & 4 & 1 & 2 & 3 & 4\end{array}$

c

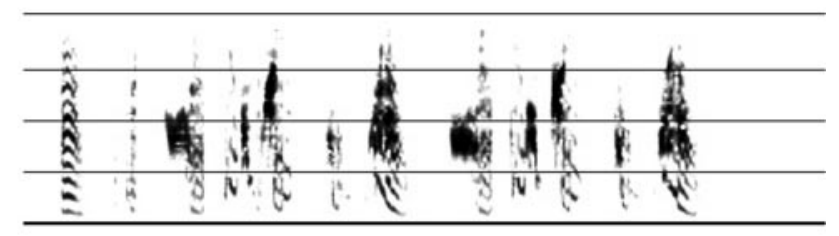

Figure 3. Two examples of songs produced before, during, and after bead implantation showing changes in overall song structure and recovery of syllable morphology. Left panel, Song before implantation $(a)$ was continuous, loud, and highly harmonic. Two weeks after bead insertion $(b)$, some syllables ( 1 and 4 ) are muted, and others ( 5 and 6 ) have been silenced, leaving long gaps in the song. After bead removal (c), the song syllables have returned to very near their prebead structure, but the missing syllables have not returned, and the temporal structure of song has changed such that syllable 1 now follows syllable 4 with a normal intersyllable interval. Right side, $a$, Prebead song; $b$, song recorded $2 \mathrm{~d}$ before bead removal. The gap left by the deletion of syllable 5 has been almost completely elided. $c$, Song recorded $6 \mathrm{~d}$ after bead removal. Syllables 1, 3, and 4 have recovered the full spectral range of the prebead song, syllable 2 has ceased to appear as a click, and syllable 4 is no longer repeated. Scale bar and axes are identical to Figure 2.

$N=36,15 ; U=224.500 ; \mathrm{NS})$. Neither initial syllable variability nor the number of dropped syllables was significantly correlated with the age of the birds. When we compared younger (142-286 d; $N=5$ ) versus older ( $>286 \mathrm{~d} ; N=4)$ birds [similar to the groups used by Lombardino and Nottebohm (2000)] for syllable variability and number of dropped syllables, we also found nonsignificant differences (initial variability: $850 \pm 19$ vs $822 \pm 40$; $U=6.000$; NS; dropped syllables: $1.0 \pm 0.5$ vs $2.5 \pm 0.5 ; U=$ 3.500 ; NS). With a larger sample size, however, age differences might have become apparent.

\section{Postbead effects}

Beads were removed 16 weeks after insertion. Within 7-9 d after removal, syllable structure was very similar to prebead structure (Figs. 3, 7, 8), except for minor differences in some syllables. To determine whether these differences were more than might be normally expected for recordings 16 weeks apart and under different recording conditions, we compared the spectrographic before and after treatment cross-correlations of bead birds to the treatment cross-correlations of sham birds. These were not significantly different (Mann-Whitney $U$ test: $N=9,5 ; U=20.500$; NS), even if we included only the seven birds that dropped syllables $(N=7,5 ; U=10.500 ; \mathrm{NS})$. Syllables did not become detectably more variable after bead treatment, because the autocorrelation scores after treatment were not lower than the pretreatment scores (Wilcoxon signed-ranks test: $N=7 ; t=7$; NS).
Summarized results for these morphological analyses appear in Table 1.

The treatment cross-correlations for both the bead and sham birds were significantly different from the initial autocorrelation scores (Wilcoxon signed-ranks tests, bead: $N=9, t=0, Z=$ 2.666, $p=0.008$; shams: $N=5, t=0, Z=2.023, p=0.043)$, which suggests that cross-correlation analysis has sufficient sensitivity to detect differences in syllables with similar morphology. To further test the sensitivity of our analysis, we compared the acrosstreatment scores from the bead birds with the control set of best different-syllable correlations constructed, as described in Materials and Methods. The treatment correlation values $(679 \pm 26)$ were significantly higher than the control set for the same birds (515 \pm 24$)$, indicating that the comparison could have detected changes in syllable morphology produced by the treatment if they had occurred (Wilcoxon signed-ranks test: $N=9, t=0, Z=$ $2.666, p=0.008$ ). Additionally, the cross-correlation scores of the distorted songs during treatment were significantly smaller than the prebead autocorrelation and the pre-post cross-correlation scores (Fig. 5) $(N=8$, prebead vs 1 month; $\mathrm{N}=9,4$ month vs postbead; both $t=0, \mathrm{Z}=-2.521, p=0.012$; there is one less bird in the early comparison because S5 was not recorded during the first 2 months of treatment).

Six of the seven birds that lost syllables while the beads were in place did not recover them (examples in Figs. 3 and $8 a$ ); the 
$\begin{array}{llllllll}1 & 2 & 3 & 4 & 5 & 6 & 7 & 8\end{array}$

a

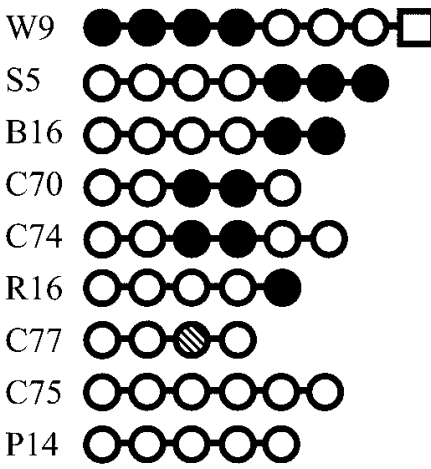

\section{b $\mathrm{Bk} 120000$ Gr20 0000000 R4 00000 B17 0000}

Figure 4. Syllables lost (and gained) during bead treatments. Open circles are syllables that were retained, filled circles are dropped syllables, the hatched symbols denote syllables that were dropped but recovered after treatment, and the square is an added syllable. The dropped syllables were contiguous in each song. $a$, Long-term bead birds. With the exception of C77, all birds permanently dropped the syllables that were silenced during bead treatment. $b$, Short-term birds. All birds recovered their deleted syllables immediately after treatment.

syllables were still missing when we recorded the birds 1 and, in some cases, 2 years later. The silent intervals from deleted syllables had become shorter by the time of bead removal in the six birds with permanently altered songs, whereas this interval was unchanged in the one bird whose dropped syllable returned (Fig. 6). In the six birds, syllable composition of the song motif did not change after the silent intervals began to shorten, although after bead removal their duration continued to decrease until they were indistinguishable from normal intersyllable intervals (Fig. 8a, last spectrogram). Bird W9 retained his added syllable in all of his post-treatment songs for at least 1 year, but the bird that began repeating a syllable during treatment (Fig. 3, right panel) did not continue to do so after bead removal.

\section{Short-term distortion}

Because permanent loss of syllables after bead treatment appeared to depend on elision of the silent intervals, we used a short-term treatment to investigate whether dropped syllables would consistently return if we removed the beads before such an elision occurred. The four birds in this group dropped one or two syllables within 4 weeks of bead insertion, leaving silent intervals of similar duration (Figs. $4 b, 8 b$ ).

Within 1 week after the song syllables were dropped, we removed the beads. In contrast to the long-term birds, the shortterm birds regained all of their deleted syllables within 1 week. All of the syllables were in the original positions in the song (Fig. $8 b)$. As in the long-term distortion experiment, we observed no apparent differences between prebead and postbead syllable morphology, but we did not perform cross-correlation analyses on the short-term birds because we had not found any changes in the long-term birds.

\section{DISCUSSION}

Long-term, reversible distortion of song in zebra finches resulted in the loss of syllables, but only minor (if any) changes in syllable

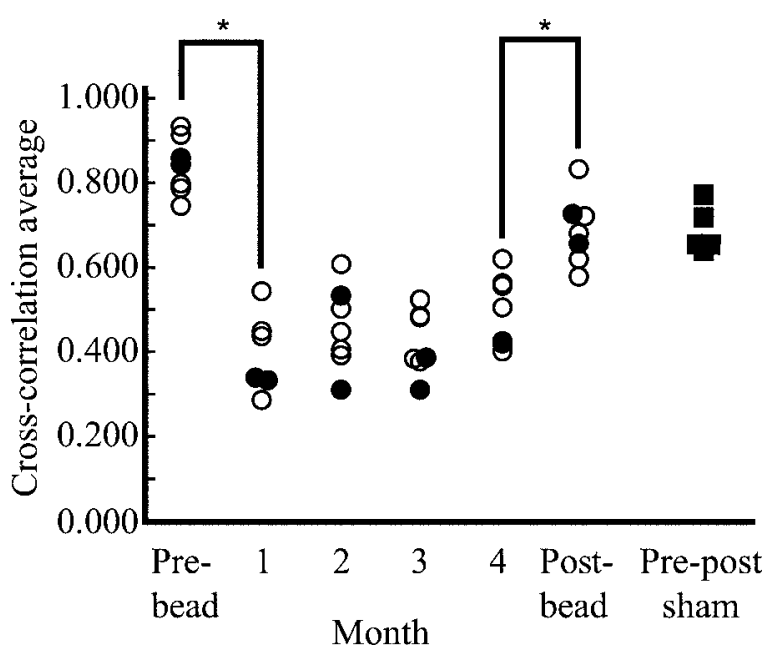

Figure 5. Cross-correlation scores between prebead songs and songs recorded during and after bead treatment, for long-term bead and sham birds. Open circles, Birds that dropped at least one syllable; solid circles, birds that did not drop syllables. Scores were averaged for each month of the treatment. The prebead value is the same-day autocorrelation for songs recorded just before bead insertion. Postbead songs were recorded between 5 and $10 \mathrm{~d}$ after bead removal, and the postbead crosscorrelation values here correspond to the treatment cross-correlation data in Table 1 . The scores at each time point were compared with those at the previous time point (Wilcoxon signed-ranks test). Significant differences (stars; $p<0.02$ ) occurred between prebead and month one, and between month four and postbead. There was no significant difference between birds that did and did not drop syllables. The solid squares show the crosscorrelation scores across the same treatment period for the sham birds, which were not significantly different from the cross-treatment scores of the bead birds (Mann-Whitney $U$ test: $N=9,5 ; U=20.500$; NS).

morphology. The altered motifs were still apparent when the birds were last recorded 1 or 2 years after treatment, and we therefore consider them permanent. In contrast, the short-term treatment did not produce any lasting changes in song.

Permanent syllable loss always co-occurred with elision of the silent interval formed when syllables were initially dropped. Although our results cannot prove that interval shortening, as opposed to the simple passage of time, caused the permanent loss, we favor the former interpretation for two reasons. First, the one long-term bird in which the gap did not shorten did recover his original motif, despite missing one syllable for 2 months. Second, two long-term birds that dropped more than one syllable still had silent intervals large enough for reinsertion of at least one syllable, yet neither recovered any deleted song elements.

It is not clear why certain syllables were dropped and others retained, although syllables in the middle and end of the motif appear most vulnerable. Deleted syllables were not necessarily the most severely distorted, nor preferentially of any syllable class. Dropped syllables were always contiguous and disappeared at the same time, to within the limits of our sampling, and may represent "chunked" syllables that form a learned unit of song in this species (Williams and Staples, 1992).

In striking contrast to the permanent loss of syllables, there was no significant change in syllable morphology by our measures. Although the same-day autocorrelations were significantly higher than the treatment correlations, we attribute this to differences in recording conditions and not to a treatment-induced change in song over time, because equal differences were observed in the bead- and sham-treated birds. Gradual changes in syllable morphology did occur during the course of treatment, which may have 


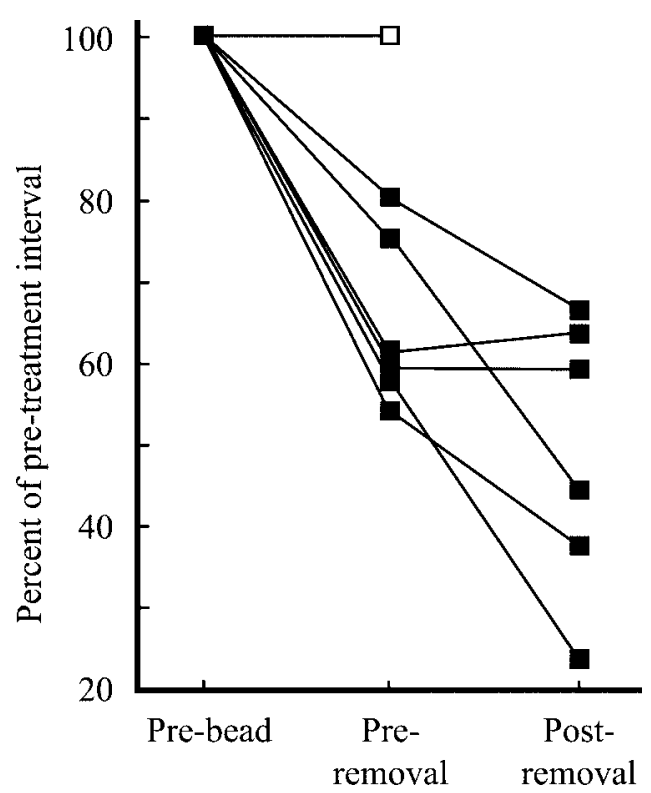

Figure 6. Duration of intersyllable interval for deleted syllables as a percentage of prebead interval. Interval duration was measured from the start of the amplitude envelope of the syllable just before a group of deleted syllables to the start of the envelope of the syllable just after the deleted group. The post-removal interval was measured 5-10 weeks after bead removal. The bird plotted with open symbols regained his dropped syllable after the bead was removed.

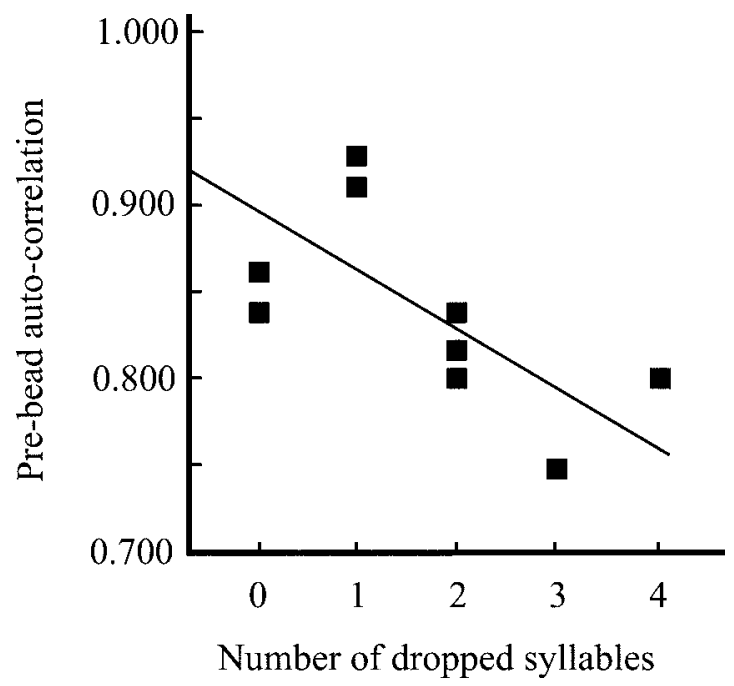

Figure 7. Plot of number of dropped syllables versus mean spectrographic autocorrelation score for songs recorded before bead implantation. Birds with more variable songs (lower autocorrelation values) dropped significantly more syllables during the 16 week distortion period. The line indicates the least-squares linear regression that best fits the data points.

resulted either from the birds actively attempting to adjust their songs or simply from changes in the position and distorting effects of the bead over time.

We propose that syllable morphology returned after bead removal because the syrinx was released from the mechanical impediment, and not through relearning of the original syllables. There was no evidence of gradual improvement with practice within the first week of recovery, beyond changes expected during air sac healing. The possibility that the birds rapidly relearned their syllables could be resolved by deafening birds at the time of removal. Regardless of the cause, it is of interest that syllable morphology, but not the missing syllables, can be recovered, as we discuss below with respect to the functional organization of the neural song control system.

\section{Comparisons with previous studies}

Other manipulations have been used to disrupt song feedback, which differ in a number of parameters: whether they affect auditory input alone or also motor output, the sounds that the birds produced during treatment, and the duration and reversibility of treatment. Although our treatment distorted song, which produced an auditory mismatch with expected auditory feedback, proprioceptive feedback may also have been altered. Although there is no evidence that the medial labia or bronchidesmus contain sensory fibers (R. Suthers, personal communication) and the beads were unlikely to have directly interfered with the syringeal muscles, proprioceptive signals might have been altered indirectly by changes in the mobility of the syringeal membranes. Bottjer and Arnold (1984) concluded that proprioception plays little role in maintaining stable song patterns in adult zebra finches, but their birds were followed for only 3 weeks.

Surgical deafening of zebra finches (Nordeen et al., 1992) and Bengalese finches (Okanoya and Yamaguchi, 1997; Woolley and Rubel, 1997) by cochlear removal causes more profound changes in song than we observed in our study. Effects of deafening include syllable degradation, syllable deletion, and loss of song stereotypy. However, in a study of Bengalese finches in which only the high-frequency hair cells were destroyed, song was completely preserved (Woolley and Rubel, 1999).

Song changes similar to those we observed (syllable loss and elision) occurred when the nXIIts nerves that innervate the syrinx were transected (Williams and McKibben, 1992). As with our technique, nerve transection produces distortion rather than a complete lack of song, and this treatment clearly alters proprioception. After the syrinx became reinnervated, changes in overall song structure stabilized, whereas the acoustic structure of distorted syllables was usually restored. With nerve transection, however, it is not possible to separate syllable retention from relearning, because reinnervation is more gradual than bead removal. In addition, improper reinnervation might also contribute to song change. Injection of botulinum toxin (a muscle paralytic) into the syringeal muscles has also been used to distort acoustic (and presumably proprioceptive) song feedback by causing motor output to resemble broadband noise bursts during treatment (Pytte and Suthers, 2000). Toxin treatment produced no permanent changes in individual syllables or syllable order after recovery. However, the distortion lasted for $<2$ months; effects more similar to ours might occur with longer treatment. The results of all of these methods are further summarized in White and Mooney (1999) and Brainard and Doupe (2000a).

Two reversible techniques that produce purely auditory interference have been used. Leonardo and Konishi (1999) observed profound changes in song motifs when they subjected birds to delayed playback of song, similar to an echo. The main result of their treatment was unstable, "decrystallized" song with many syllable repetitions and some syllables that were rarely sung, but never completely discarded. After normal feedback was restored, song gradually returned. However, in all birds, the original song motif was occasionally sung throughout the treatment. After normal feedback was restored, song gradually returned, perhaps recapitulating the process of song crystallization during initial 
Figure 8. Examples of syllable deletion and interval elision in a long-term and a short-term bead bird. Vertical lines separate prebead, bead, and postbead spectrograms. In the longterm bird $(a)$, the initial duration of the interval between the onsets of syllables 4 and 1 was $294 \mathrm{msec}$. Syllable 5 was highly distorted during the first month of treatment (second spectrogram, $317 \mathrm{msec}$ intersyllable interval), and was dropped during the third month (third spectrogram, $272 \mathrm{msec}$ interval). By the time a of bead removal, the interval was somewhat shortened; $(195 \mathrm{msec})$, and it continued to close until it reached normal syllable spacing 2 months after treatment $(176 \mathrm{msec})$. Recordings were at 1 week before treatment, week 2 and 15 of treatment, and 1 week and 6 weeks after treatment. In the short-term bird $(b)$, the pretreatment interval between the onsets of syllables 4 and 7 was 434 msec. When syllables 5 and 6 were dropped during the second week of treatment, the gap did not shorten (second spectrogram, $445 \mathrm{msec}$ interval), and the syllables returned 1 week after the bead was removed (424 msec interval). Recordings were at 1 week before treatment, week 2 of treat- b ment, and 1 week after treatment. Treatment durations are as specified in Materials and Methods. Scale bar and axes are identical to Figure 2.

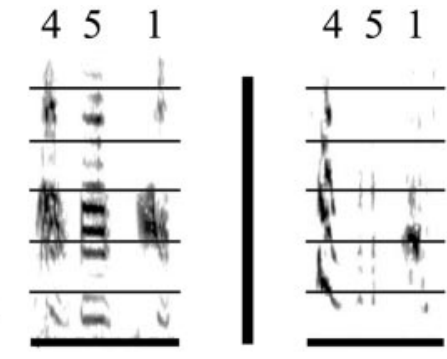

Pre-bead

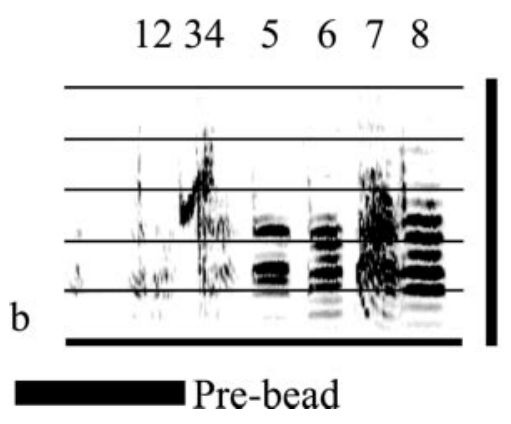

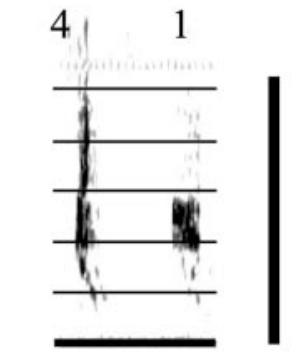

Bead
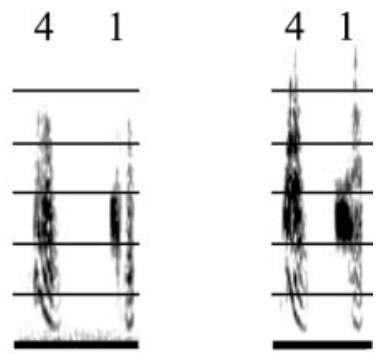

Post-bead

Table 1. Spectrogram cross-correlation values for long-term bead birds and sham treatment birds

\begin{tabular}{|c|c|c|c|c|c|}
\hline Group & $N$ & $\begin{array}{l}\text { Pre-bead } \\
\text { autocorrelation }\end{array}$ & $\begin{array}{l}\text { Treatment } \\
\text { cross-correlation }\end{array}$ & $\begin{array}{l}\text { Post-bead } \\
\text { autocorrelation }\end{array}$ & $\begin{array}{l}\text { Deleted } \\
\text { syllables }\end{array}$ \\
\hline Bead & 9 & $837 \pm 26^{a}$ & $679 \pm 25$ & $801 \pm 21$ & $15 / 51(29 \%)$ \\
\hline Bead-D ${ }^{b}$ & 7 & $837 \pm 30$ & $672 \pm 39$ & $801 \pm 24$ & \\
\hline Sham & 5 & $798 \pm 28$ & $685 \pm 23$ & $823 \pm 17$ & $00 / 25(0 \%)$ \\
\hline
\end{tabular}

${ }^{a}$ Correlations range between 0 (no similarity) and 1000 (identical). Values are mean \pm SEM.

${ }^{b}$ This group includes only birds that deleted syllables during bead treatment.

song learning. Zevin et al. (2000) subjected birds to high levels of white noise for weeks. Birds that were allowed to hear their own songs for only $1 \mathrm{hr}$ each week had remarkably preserved songs, whereas the songs of birds with no opportunity for normal feedback were disrupted to varying degrees. After the noise treatment was stopped, the permanency of song change correlated with the extent of song disruption during treatment, but the nature of the permanent changes, and the time course of recovery when it occurred, have not yet been described.

A suggestion consistent with all the results cited above is that correct temporal signals provided by song feedback may be sufficient to maintain the song motor program, even when the acoustic content of these signals is abnormal. A sustaining role for temporal input would explain why our birds recovered severely dampened or distorted elements. Woolley and Rubel (1999) also suggested that temporal cues may have maintained song in their partially deafened Bengalese finches, but they could not distinguish between temporal cues as opposed to low-frequency spectral cues. The importance of correct temporal cues is consistent with the more severe degradation seen when birds hear delayed feedback, because this treatment destroys timing cues (Leonardo and Konishi, 1999). For a review on the importance of timing signals for the production of song, see Konishi (1994).

\section{Implications for neural mechanisms involved in adult song maintenance and plasticity}

The effects produced by different methods of perturbing song feedback suggest hypotheses about the role of specific neural pathways in maintaining the song motor program. First, it should be noted that the results of several studies, considered together, argue against central neural degeneration as the cause of deafening-induced song degradation. Song is preserved even when most of the hair cells in the cochlea are destroyed (Woolley and Rubel, 1999) and when deafening is combined with lesions in the anterior forebrain pathway (specifically, nucleus LMAN) of the neural song system (Brainard and Doupe, 2000b). This latter experiment, and a similar one in which LMAN lesions were combined with nerve transection (Williams and Mehta, 1999), indicate that song changes produced by perturbation of feedback require either active or permissive signals in the anterior forebrain pathway. In addition, noninvasive methods can severely affect song (Leonardo and Konishi, 1999; Zevin et al., 2000). Thus, is it unlikely that the changes in song produced by any methods that have been used are merely a passive result of neural degeneration.

Song motor control is hierarchically organized in the forebrain: nucleus $\mathrm{HVc}$ connects to the robust nucleus of the archistriatum (RA), which innervates the motor neurons of $\mathrm{nXIIts}$ that coordi- 
nate the syringeal muscles (Nottebohm et al., 1976). Electrophysiological activity recorded during singing (McCasland and Konishi, 1981; McCasland, 1987; Yu and Margoliash, 1996) and electrical stimulation experiments ( Vu et al., 1994; Vicario and Simpson, 1995) indicate that syllable sequencing and the overall temporal pattern of song is encoded within, or upstream from, $\mathrm{HVc}$, whereas individual syllables are organized within RA (for review, see Vicario, 1994; Margoliash, 1997). The anterior forebrain pathway (AFP) forms a separate, longer pathway from HVc to RA (Nottebohm et al., 1976; Bottjer et al., 1989, 2000), and neurons in this pathway are also active during singing (Hessler and Doupe, 1999).

Neurons in HVc (Margoliash, 1983, 1986) and the AFP (Doupe, 1997) respond selectively to a bird's own song. These auditory responses may serve to reinforce song as it is performed, even when song syllables are abnormal. Although song system neurons would not be expected to fire maximally to distorted song, HVc neurons do retain some responsiveness to spectrally degraded song presented in the correct temporal pattern (Fortune and Margoliash, 1992; Theunissen and Doupe, 1998). Auditory responsive neurons that project to $\mathrm{HVc}$, either directly or indirectly, have less selective song responses (Stripling et al., 1997; Janata and Margoliash, 1999; Mooney 2000) and might also be involved in song maintenance.

The permanent alteration in motif structure coupled with the rapid return of syllable morphology that we observed suggest that only the motor program in the song circuit upstream from RA (including possibly the afferents to RA) was permanently changed. The initial deletion of syllables may reflect an inability of $\mathrm{HVc}$ to sufficiently stimulate RA neurons encoding those syllables, even while the song motor pattern remains intact at higher levels. Loss of contact might occur more easily in birds where the connections are less firmly established, as evidenced by the correlation between pretreatment syllable variability and the number of elements lost (Fig. 7). Connection loss within RA could then retrogradely trigger reorganization of the motor program at higher levels. Eventually, the program for the motif would be "patched" as nonreinforced portions were removed, resulting in permanent change. Once the new pattern has been established, it would be actively reinforced and prevent reversion to the old pattern.

The above scenario raises at least three questions about which we can only speculate: (1) why would deleted syllables return after short-term bead treatment? Perhaps the loss of drive between $\mathrm{HVc}$ and RA is incomplete at early stages, and connections are re-established based on activity produced by the robust return of adjacent song syllables, as long as the original motor program is still instantiated within the system and has not been edited. (2) Why are altered or new syllables produced with treatments such as deafening or delayed feedback? When correct temporal cues are missing entirely, fewer of the original connections to RA may be preserved. Then new connections might be more likely to form, perhaps involving new projection neurons from $\mathrm{HVc}$ to RA (Kirn and Nottebohm, 1993; Scharff et al., 2000; Scott et al., 2000), to produce syllables different from those in the original song. (3) If reorganization of the motor program occurs upstream from RA, how can lesions of LMAN (which projects to RA, but has no direct access to $\mathrm{HVc}$ ) preserve song (Williams and Mehta, 1999; Brainard and Doupe, 2000b)? One possible explanation is that LMAN provides the signals required for the initial "release" of HVc connections from RA.

Our technique for disrupting song feedback enables investigation of specific questions about the neural mechanisms of adult song reorganization because it is reversible and produces predictable changes in song at different times during treatment. For example, it would be intriguing to examine whether the selective auditory responses present in song system nuclei change after portions of song are lost, because this selectivity is known to emerge during song development under the guidance of song feedback (Volman, 1993; Solis and Doupe, 1997, 1999, 2000). Our results add to those of other studies on the importance of correct song feedback, collectively providing an emerging picture of the mechanisms underlying song maintenance, and the limits of song plasticity, in adult songbirds.

\section{REFERENCES}

Bottjer SW, Arnold AP (1984) The role of feedback from the vocal organ. I. Maintenance of stereotypical vocalizations by adult zebra finches. J Neurosci 4:2387-2396.

Bottjer SW, Halsema KA, Brown SA, Miesner EA (1989) Axonal connections of a forebrain nucleus involved with vocal learning in zebra finches. J Comp Neurol 279:312-326.

Bottjer SW, Brady JD, Cribbs B (2000) Connections of a motor cortical region in zebra finches: relation to pathways for vocal learning. J Comp Neurol 420:244-260.

Brainard MS, Doupe AJ (2000a) Auditory feedback in learning and maintenance of vocal behaviour. Nat Rev Neurosci 1:31-40.

Brainard MS, Doupe AJ (2000b) Interruption of a basal gangliaforebrain circuit prevents plasticity of learned vocalizations. Nature 404:762-766.

Doupe AJ (1997) Song- and order-selective neurons in the songbird anterior forebrain and their emergence during vocal development. J Neurosci 17:1147-1167.

Fortune ES, Margoliash D (1992) Cytoarchitectonic organization and morphology of cells of the field L complex in male zebra finches (Taeniopygia guttata). J Comp Neurol 325:388-404.

Goller F, Larsen ON (1997) A new mechanism of sound generation in songbirds. Proc Natl Acad Sci USA 94:14787-14791.

Hessler NA, Doupe AJ (1999) Singing-related neural activity in a dorsal forebrain-basal ganglia circuit of adult zebra finches. J Neurosci 19:10461-10481.

Hough II GE, Volman SF (1996) Long-term effects of song perturbation on song production in zebra finches. Soc Neurosci Abstr 22:963.

Hough II GE, Volman SF (2000) Re-expression of songs deleted during vocal development in white-crowned sparrows, Zonotrichia leucophrys. Anim Behav 60:279-287.

Janata P, Margoliash D (1999) Gradual emergence of song selectivity in sensorimotor structures of the male zebra finch song system. J Neurosci 19:5108-5118.

Janik VM (1999) Pitfalls in the categorization of behaviour: a comparison of dolphin whistle classification methods. Anim Behav 57:133-143.

Khanna H, Gaunt SLL, McCallum DA (1997) Digital spectrographic cross-correlation: tests of sensitivity. Bioacoustics 7:209-234.

Kirn JR, Nottebohm F (1993) Direct evidence for loss and replacement of projection neurons in adult canary brain. J Neurosci 13:1654-1663.

Konishi M (1965) The role of auditory feedback in the control of vocalization in the white-crowned sparrow. Z Tierpsychol 22:770-783.

Konishi M (1994) Pattern generation in birdsong. Curr Opin Neurobiol 4:827-831.

Leonardo A, Konishi M (1999) Decrystallization of adult birdsong by perturbation of auditory feedback. Nature 399:466-470.

Lombardino AJ, Nottebohm F (2000) Age at deafening affects the stability of learned song in adult male zebra finches. J Neurosci 20:5054-5064.

Margoliash D (1983) Acoustic parameters underlying the responses of song specific neurons in the white-crowned sparrow. J Neurosci 3:1039.

Margoliash D (1986) Preference for autogenous song by auditory neurons in a song system nucleus of the white-crowned sparrow. J Neurosci 6:1643-1661.

Margoliash D (1997) Functional organization of forebrain pathways for song production and perception. J Neurobiol 33:671-693.

Marler P (1970) A comparative approach to vocal learning: song development in white-crowned sparrows. J Comp Physiol Psychol 71:1-25.

McCasland JS (1987) Neuronal control of bird song production. J Neurosci 7:23-39.

McCasland JS, Konishi M (1981) Interaction between auditory and motor activities in an avian song control nucleus. Proc Natl Acad Sci USA 78:7815-7819.

McDonald MV (1989) Function of song in Scott's Seaside Sparrow, Ammodramus maritimus peninsulae. Anim Behav 38:468-487.

Mooney R (2000) Different subthreshold mechanisms underlie song selectivity in identified $\mathrm{HVc}$ neurons of the zebra finch. J Neurosci 20:5420-5436. 
Nordeen KW, Nordeen EJ (1992) Auditory feedback is necessary for the maintenance of stereotyped song in adult zebra finches. Behav Neural Biol 57:58-66.

Nordeen EJ, Grace A, Burek MJ, Nordeen KW (1992) Sex-dependent loss of projection neurons involved in avian song learning. J Neurobiol 23:671-679.

Nottebohm F (1968) Auditory experience and song development in the Chaffinch (Fringilla coelebs): ontogeny of a complex motor pattern. Ibis 110:549-568.

Nottebohm F, Stokes TM, Leonard CM (1976) Central control of song in the canary, Serinus canarius. J Comp Neurol 165:457-486.

Okanoya K, Yamaguchi A (1997) Adult Bengalese finches (Lonchura striata var. domestica) require real-time auditory feedback to produce normal song syntax. J Neurobiol 33:343-356.

Price PH (1979) Developmental determinants of structure in zebra finch songs. J Comp Physiol Psychol 93:260-277.

Pytte CL, Suthers RA (2000) Sensitive period for sensorimotor integration during vocal motor learning. J Neurobiol 42:172-189.

Scharff C, Kirn JR, Grossman M, Macklis JD, Nottebohm F (2000) Targeted neuronal death affects neuronal replacement and vocal behavior in adult songbirds. Neuron 25:481-492.

Scott LL, Nordeen EJ, Nordeen KW (2000) The relationship between rates of HVc neuron addition and vocal plasticity in adult songbirds. J Neurobiol 43:79-88.

Smith DG (1979) Male singing ability and territory integrity in redwinged blackbirds. Behaviour 68:193-206.

Solis MM, Doupe AJ (1997) Anterior forebrain neurons develop selectivity by an intermediate stage of birdsong learning. J Neurosci 17:6447-6462.

Solis MM, Doupe AJ (1999) Contributions of tutor and bird's own song experience to neural selectivity in the songbird anterior forebrain. J Neurosci 19:4559-4584.

Solis MM, Doupe AJ (2000) Compromised neural selectivity for song in birds with impaired sensorimotor learning. Neuron 25:109-121.

Sossinka R, Böhner J (1980) Song types in the zebra finch Poephila guttata castanotis. Z Tierpsychol 53:123-132.

Stripling R, Volman SF, Clayton DF (1997) Response modulation in the zebra finch neostriatum: relationship to nuclear gene regulation. J Neurosci 17:3883-3893.

Sturdy CB, Phillmore LS, Weisman RG (1999) Note types, harmonic structure, and note order in the songs of zebra finches (Taeniopygia guttata). J Comp Psychol 113:194-203.
Suthers RA, Goller F, Pytte C (1999) The neuromuscular control of birdsong. Philos Trans R Soc Lond B Biol Sci 354:927-939.

Tchernichovski O, Nottebohm F, Ho CE, Pesaran B, Mitra PP (2000) A procedure for an automated measurement of song similarity. Anim Behav 59:1167-1176.

Theunissen FE, Doupe AJ (1998) Temporal and spectral sensitivity of complex auditory neurons in the nucleus HVc of male zebra finches. J Neurosci 18:3786-3802.

Tramontin AD, Brenowitz EA (2000) Seasonal plasticity in the adult brain. Trends Neurosci 23:251-258.

Vicario DS (1994) Motor mechanisms relevant to auditory-vocal interactions in songbirds. Brain Behav Evol 44:265-278.

Vicario DS, Simpson HB (1995) Electrical stimulation in forebrain nuclei elicits learned vocal patterns in songbirds. J Neurophysiol 73:2602-2607.

Volman SF (1993) Development of neural selectivity for birdsong during vocal learning. J Neurosci 13:4737-4747.

Vu ET, Mazurek ME, Kuo YC (1994) Identification of a forebrain motor programming network for the learned song of zebra finches. J Neurosci 14:6924-6934.

Wang N, Aviram R, Kirn JR (1999) Deafening alters neuron turnover within the telencephalic motor pathway for song control in adult zebra finches. J Neurosci 19:10554-10561.

White SA, Mooney R (1999) Can an old bird change his tune? Curr Biol 9:R688-R690.

Williams H, McKibben JR (1992) Changes in stereotyped central motor patterns controlling vocalization are induced by peripheral nerve injury. Behav Neural Biol 57:67-78.

Williams H, Mehta N (1999) Changes in adult zebra finch song require a forebrain nucleus that is not necessary for song production. J Neurobiol 39:14-28.

Williams H, Staples K (1992) Syllable chunking in zebra finch (Taeniopygia guttata) song. J Comp Psychol 106:278-286.

Woolley SM, Rubel EW (1997) Bengalese finches Lonchura Striata domestica depend upon auditory feedback for the maintenance of adult song. J Neurosci 17:6380-6390.

Woolley SM, Rubel EW (1999) High-frequency auditory feedback is not required for adult song maintenance in Bengalese finches. J Neurosci 19:358-371.

Yu AC, Margoliash D (1996) Temporal hierarchical control of singing in birds. Science 273:1871-1875.

Zevin JD, Seidenberg MS, Bottjer SW (2000) Song plasticity in adult zebra finches exposed to white noise. Soc Neurosci Abstr 26:723. 